\title{
Spiral Colon
}

National Cancer Institute

\section{Source}

National Cancer Institute. Spiral Colon. NCI Thesaurus. Code C92438.

The ascending colon of the ruminants and pigs. 